\title{
Tension Pneumothorax as Initial Manifestation of Granulomatosis with Polyangiitis (GPA)
}

\author{
Rúben Reis, Anneke Joosten, Francelino Ferreira, Magda Silva, Catarina Parente, Inês Maia \\ Internal Medicine Department, Centro Hospitalar Barreiro-Montijo, Barreiro, Portugal
}

Received: $17 / 01 / 2021$

Accepted: $26 / 01 / 2021$

Published: $11 / 02 / 2021$

\begin{abstract}
How to cite this article: Reis R, Joosten A, Ferreira F, Silva M, Parente C, Maia I. Tension pneumothorax as initial manifestation of granulomatosis with polyangiitis (GPA). EJCRIM 2021;8: doi:10.12890/2021_002304.
\end{abstract}

Conflicts of Interests: The Authors declare that there are no competing interests.

This article is licensed under a Commons Attribution Non-Commercial 4.0 License

\section{ABSTRACT}

Granulomatosis with polyangiitis (Wegener's granulomatosis) is a systemic vasculitis that primarily affects small and medium vessels. Its manifestations are usually confined to the upper airway, lower airway and kidney. It can also affect other organs and systems, although this is unusual. We describe the case of a 67-year-old woman who presented with a tension pneumothorax due to rupture of a pulmonary cavity. This pulmonary cavity proved to be secondary to systemic disease which also caused a tumour in her kidney. Biopsy showed nonnecrotizing granulomatosis, and even though antineutrophil cytoplasmic antibodies (ANCA) were negative, the diagnosis of granulomatosis with polyangiitis was made.

\section{LEARNING POINTS}

- Granulomatosis with polyangiitis (GPA) can be a challenging diagnosis when the initial manifestation is atypical, so a careful history and physical examination are needed to make the diagnosis.

- It is not uncommon for patients with multisystemic inflammatory disease to attend several different specialty clinics before the diagnosis is reached.

- GPA with negative ANCA is rare, and occurs more frequently in non-severe forms of the disease.

\section{KEYWORDS}

Granulomatosis with polyangiitis (Wegener's), double ANCA negative, tension pneumothorax, cavitation, renal mass

\section{CASE DESCRIPTION}

We present the case of a 67-year-old woman admitted to the emergency ward with acute dyspnoea and pleuritic pain in the right hemithorax. Her vital signs were as follows: blood pressure $80 / 40 \mathrm{mmHg}$, heart rate 140 beats per minute, rhythmic, febrile with a temperature of $39.4^{\circ} \mathrm{C}$, polypnoea with 25 breaths per minute and peripheral oxygen saturation of $90 \%$. Upon thoracic examination, she had absent lung sounds on the right hemithorax and hyper-resonance. A chest x-ray confirmed the presence of a tension pneumothorax and upper lobe cavities bilaterally (Fig. 1). Further investigation revealed the patient had previously been admitted years before due to self-limiting paraparesis. She was also attending an otolaryngology clinic because of chronic rhinosinusitis with multiple poorly defined lesions in her nasopharynx, and a pneumology clinic regarding bilateral pulmonary cavities. She was not on any chronic medication at this time.

The patient reported being unwell for the past 3 months. During this period, she unwillingly lost $25 \mathrm{~kg}$, was experiencing anorexia, felt weak and had a persistent cough. She also reported persistent fever mainly during the late afternoon until night time, ranging from $38^{\circ} \mathrm{C}$ to $39.5^{\circ} \mathrm{C}$. Multiple courses of antibiotics were completed during this time but the fever, malaise and weight loss persisted. After stabilization, the patient was admitted to the medicine ward for further investigation. 


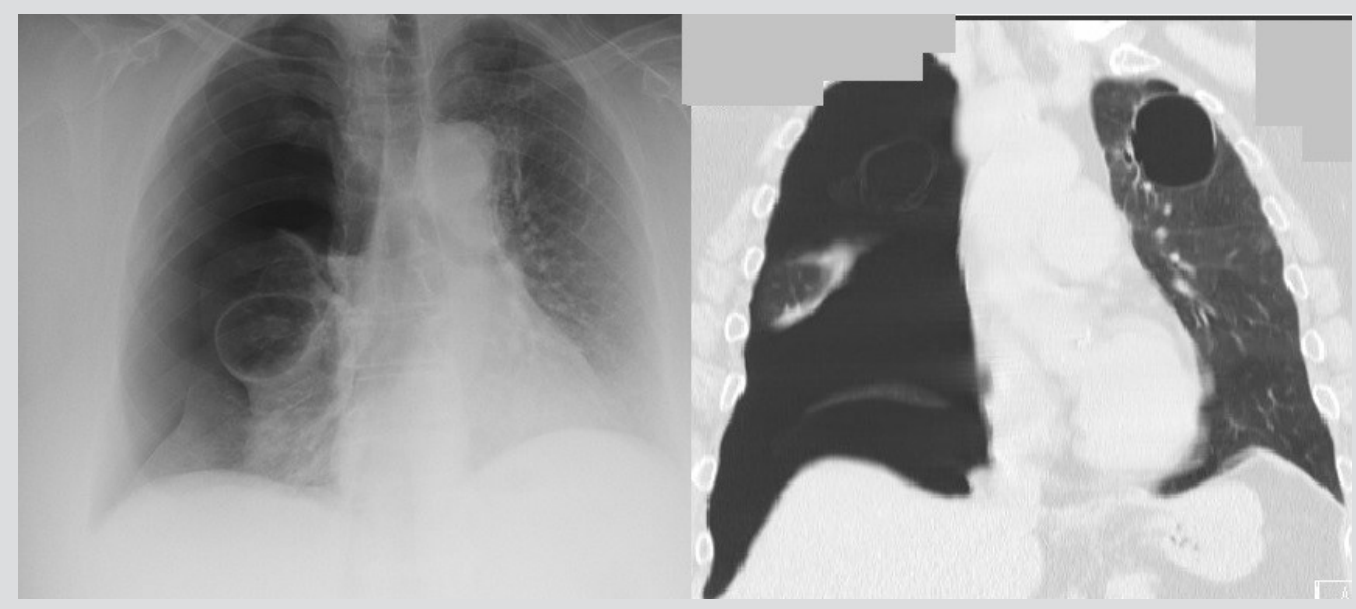

Figure 1. X-ray of the right pneumothorax and CT of the thorax

The patient's initial laboratory evaluation showed anaemia ( $8.3 \mathrm{~g} / \mathrm{dl}$ ), high C-reactive-protein (193.9 mg/dl) and a sedimentation rate of 120 $\mathrm{mm}$. Serum creatinine and blood urea nitrogen were normal. Urinalysis was normal. Laboratory findings are presented in Table 1. Blood, urine and sputum cultures were negative for pathogenic microorganisms and full body computed tomography was requested. In addition to the numerous, previously known pulmonary cavities (Fig. 2), a renal mass was also seen (Fig. 3).
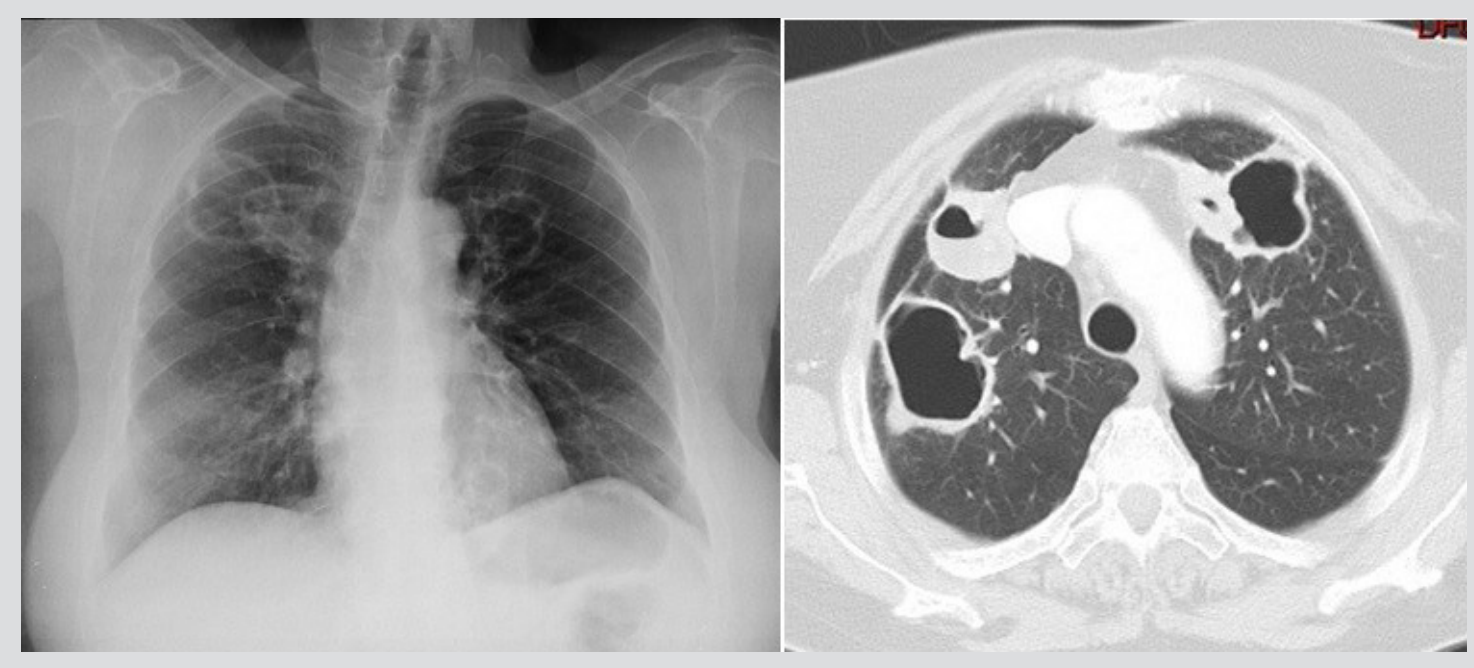

Figure 2.. X-ray of lung cavities and CT of the thorax

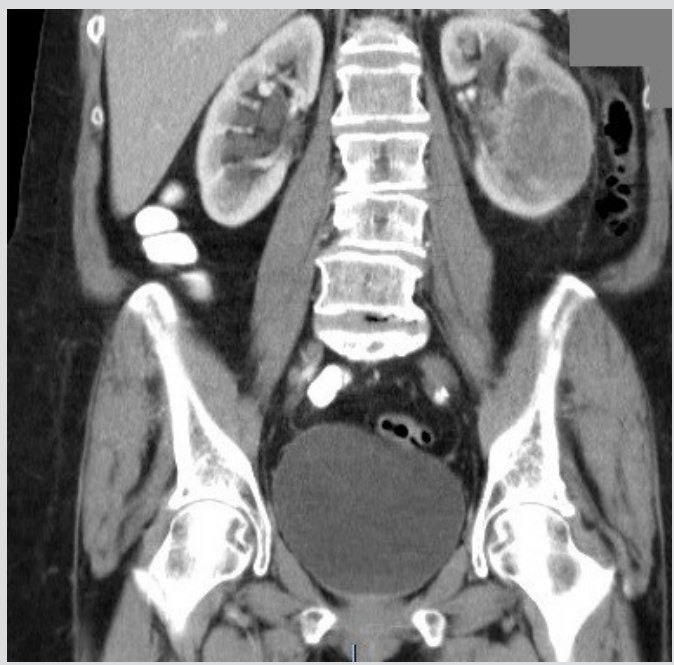

Figure 3. Abdominal CT showing left renal mass 


\begin{tabular}{|l|l|}
\hline Parameter & Value \\
\hline Haemoglobin & $8.6 \mathrm{~g} / \mathrm{dl}$ \\
\hline Leucocytes & $9.2 \times 10^{9} / \mathrm{l}$ \\
\hline Neutrophils & $73.6 \%$ \\
\hline Lymphocytes & $16.5 \%$ \\
\hline Platelet count & $384 \times 10^{9} / \mathrm{l}$ \\
\hline Serum creatinine & $0.87 \mathrm{mg} / \mathrm{dl}$ \\
\hline BUN & $26 \mathrm{mg} / \mathrm{dl}$ \\
\hline Antinuclear antibodies & Negative \\
\hline PR3-ANCA & Negative \\
\hline MPO-ANCA & Negative \\
\hline C-reactive protein & $193.9 \mathrm{mg} / \mathrm{dl}$ \\
\hline Sedimentation rate & $>120 \mathrm{~mm}$ \\
\hline Urinalysis & Normal \\
\hline
\end{tabular}

A renal biopsy was performed, revealing a granulomatous inflammatory process with incomplete central necrosis. The characteristics were atypical for tuberculosis and no microorganisms were identified. Observation by an otolaryngologist was requested, who reported a hyperkeratotic nasal mass. Bronchofibroscopy was also performed and bronchiolar lavage was requested but did not yield conclusive results (uncharacteristic inflammatory infiltrate). Antinuclear antibody tests, as well as for other antibodies such as ANCA, were requested and came back negative. Before initial therapy, a search for other complications was performed. MRI excluded brain and eye involvement. After tuberculosis and other infectious diseases were excluded, granulomatosis with polyangiitis (GPA) with negative ANCA was diagnosed and the patient was started on therapy. As involvement was limited to the upper and lower airway (no glomerulonephritis was present), the initial approach to achieve remission was to use high-dose steroid (methylprednisolone $1 \mathrm{~g}$ for 3 days) and methotrexate $15 \mathrm{mg}$. The patient's inflammatory parameters reduced as did her body temperature. She was discharged after 5 days of improvement on $1 \mathrm{mg} / \mathrm{kg}$ prednisolone and $15 \mathrm{mg}$ methotrexate. Six months after discharge she had maintained clinical remission with methotrexate and low-dose prednisolone.

\section{DISCUSSION}

GPA (Wegener's granulomatosis) is a rare condition, with an annual incidence of 2.4-11.3 per million. It mainly affects the upper respiratory tract ( $90 \%$ of patients), lungs and kidneys, but may also affect the eyes, central nervous system, gastrointestinal tract, skin and musculoskeletal system ${ }^{[1]}$. Spontaneous pneumothorax is estimated to occur in 3-5\% of all GPA patients, although only a few such cases have actually been reported. The majority of affected patients are male (>70\%) and one review article reported a mortality rate of approximately $40 \%$. Conditions described range from small subpleural pneumothorax to tension pneumothorax, pyopneumothorax and hydropneumothorax. The cause of pneumothorax in GPA is still unclear, although it is hypothesized to be related to a subpleural fistula and/or cavity dimension (usually $>20 \mathrm{~mm})^{[2,3]}$. The diagnosis is usually based on the presence of ANCA markers as they are found in most patients with GPA. PR3ANCA is positive in up to $87.1 \%$ and MPO-ANCA in $14.7 \%$ of patients. Nevertheless, a negative ANCA essay does not mean GPA should be excluded, as the milder forms of the disease (that only affect the upper respiratory tract) are more commonly associated with negative ANCA (96\% in severe disease compared with $83 \%$ in milder/limited disease) ${ }^{[4]}$.

Review of this particular case reveals the patient showed multiple manifestations that can now be attributed to GPA. These include the findings in her upper and lower respiratory tract, her previous admission to the neurology ward, her skin lesions and fever, laboratory alterations and renal mass. The reason for admission, which ultimately initiated diagnostic confirmation by review CT, was the suspected pulmonary cavity which triggered the pneumothorax and which was found to be apical, subpleural and about $25 \mathrm{~mm}$ in diameter, characteristics that may increase the risk of pneumothorax ${ }^{[3]}$.

Even though the ANCA markers were negative, the American College of Rheumatology diagnostic criteria were met, as the patient had nasal inflammation, abnormal chest radiography and granulomatous inflammation. The only criterion she did not meet was abnormal urinary sediment. These criteria have a sensitivity of $88 \%$ and a specificity of $92 \%$ for GPA ${ }^{[5]}$. 


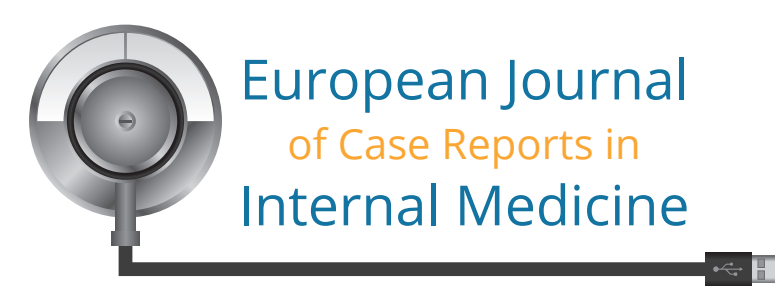

The therapeutic approach should depend on the severity of the disease. Rituximab or cyclophosphamide should be considered for severe disease, while methotrexate can be useful for mild to moderate disease. Steroids should be used as adjunct therapy and tapered after remission is achieved. After remission, azathioprine, methotrexate and leflunomide can be options to maintain remission. Rituximab is also being used with great benefit as maintenance therapy in serious cases ${ }^{[5]}$.

\section{REFERENCES}

1. Grygiel-Górniak B, Limphaibool N, Perkowska K, Puszczewicz M. Clinical manifestations of granulomatosis with polyangiitis: key considerations and major features. Postgrad Med 2018;130(7):581-596.

2. Belhassen-Garcia M, Velasco-Tirado V, Alvela-Suaréz L, Carpio-Pérez A, Lledías JP, Novoa N, et al. Spontaneous pneumothorax in Wegener's granulomatosis: case report and literature review. Semin Arthritis Rheum 2011;41(3):455-460.

3. Kumar S, Pawani N, Bawankule S, Diwan S, Honmode A, Jain S. Wegener's granulomatosis presenting as spontaneous pneumothorax in young adult. J Assoc Chest Physicians 2014;2(2):75.

4. Kashiwagi T, Hayama N, Fujita E, Hara K, Mii A, Masuda Y, et al. A case of (double) ANCA-negative granulomatosis with polyangiitis (Wegener's). CEN Case Rep 2012;1(2):104111.

5. Lutalo PMK, D'Cruz DP. Diagnosis and classification of granulomatosis with polyangiitis (aka Wegener's granulomatosis). J Autoimmun 2014;48-49:94-98. 